\section{Moderne chirurgische Behandlung von Lebermetastasen}

\author{
H. U. Baer, C. Kulli, E. X. Jensen
}

\begin{abstract}
Einleitung
In den letzten zwanzig Jahren hat sich die Behandlung von Lebermetastasen stark gewandelt. Galt die Leber früher als ein Organ, das chirurgisch nicht berührt werden sollte, so werden heute ausgedehnte komplexe Leberresektionen mit kombinierten onkologischen Therapien an spezialisierten Zentren von hepatobiliär ausgebildeten Chirurgen und Onkologen erfolgreich mit einer geringen Morbidität und Letalität durchgeführt. Es ist heute unbestritten, dass die besten Resultate für Lebermetastasen erreicht werden, wenn die möglichen Therapieformen interdisziplinär besprochen und eingesetzt werden. Die guten Resultate der chirurgischen Behandlungen, die in den letzen zehn Jahren erreicht werden konnten, waren nur möglich dank Fortschritten auf den Gebieten der chirurgischen Technik, der präoperativen radiologischen und hepatologischen Abklärung, der Anästhesie und postoperativen Intensivmedizin sowie dem wachsenden Verständnis der schwierigen chirurgischen Leberanatomie mit ihren zahllosen Varianten (Tab. 1).

Kolorektale Lebermetastasen sind häufig und werden zum Zeitpunkt der primären Kolonresektion in 10-25\% gefunden [1]. Nur etwa 25\% der nachgewiesenen kolorektalen Lebermetastasen wurden bis anhin als resektabel angesehen [2]. Oft fiel bisher eine Resektion ausser Betracht, weil die Metastasen diffus oder multifokal in der Leber verteilt waren, zu wenig funktionierendes Lebergewebe nach einer ausgedehnten Resektion übriggeblieben wäre oder weil die Resektion von mehr als 4-5 Metastasen aus prognostischen Gründen als unsinnig erschien. Bei fortschreitendem kolorektalen Karzinomleiden ist die Leber aber in 40-70\% mitbetroffen und in bis zu 30\% bleibt sie sogar der einzige Ort der Metastasierung [3]. Die komplette chirurgische Resektion aller Metastasen bleibt für diese Patientinnen und Patienten aber bis heute die einzige Hoffnung auf eine möglicherweise kurative Behandlung. Ob neue Chemotherapeutika, die in der nächsten Zeit klinisch eingeführt
\end{abstract}

Korrespondenz:

Prof. Dr. med. Hans U. Baer

Klinik Hirslanden

GastroZentrum, Viszeralchirurgie

Witellikerstrasse 40

CH-8029 Zürich werden, diese Perspektiven verbessern, bleibt abzuwarten. Für nicht behandelte kolorektale Lebermetastasen ist die Prognose bekannterweise schlecht. Die mediane Überlebenszeit beträgt nur 2 Jahre und nur vereinzelte Patienten überleben 5 Jahre [4].

Es ist deshalb seit Jahren ein dringendes Anliegen der Leberchirurgen, dass auf der einen Seite Patienten mit möglicherweise resektablen Metastasen auch wirklich zur Operation zugewiesen werden, auf der anderen Seite Behandlungsmethoden eingeführt werden, die das therapeutische chirurgische Spektrum erweitern und die Überlebenschancen der Betroffenen mitverbessern.

In den letzten Jahren sind die bisher standardisierten Indikationen zur Leberresektion plötzlich etwas überholt worden, weil neu eingeführte Methoden der Hitze-, Kälte- und Lasertherapie (Tab. 2) einige Veränderungen und auch Erweiterungen des heutigen Indikationsspektrums für die Behandlung von Lebermetastasen gebracht haben.

Diese Arbeit analysiert kurz die Resultate der konventionellen resezierenden Chirurgie (für die am häufigsten auftretenden kolorektalen Lebermetastasen) und versucht aufzuzeigen, wo heute neu Indikationen zu einer kombinierten Behandlung von Lebermetastasen bestehen und welche neuen teils chirurgisch, teils interventionell einsetzbaren Verfahren die Indikationsstellung bereits verändert haben.

\section{Chirurgische Standardtherapie und ihre Resultate}

Aus chirurgischer Sicht scheint es sinnvoll, zwischen Operabilität und Resektabilität zu unterscheiden. Die Operabilität wird dabei definiert als der Allgemeinzustand des Patienten im Hinblick auf die Grösse der Operation. Die präoperative Abklärung der Operabilität umfasst deshalb Allgemeinstatus, Ausschluss eines Lokalrezidivs, Belastungs-EKG, Lungenfunktionsprüfung, CT oder MRI des Thorax. Die Resektabilität bezieht sich auf die technische Möglichkeit, eine Leberresektion (im Gesunden) durchzuführen. Die präoperative Abklärung der Resektabilität umfasst native und Kontrastmitteluntersuchung der Leber mit (Spiral-)CT oder MRI der Leber mit Kontrastmittel, eventuell 3D-Rekonstruktion, eventuell ein PET-Szintigramm, sowie die Untersuchung der Leberfunktion mit Laboruntersuchung, Suche nach Tumormarkern und Leberfunktionstests wie die GEK (Galaktose-Eliminationskapazität). Die chirurgische präoperative Grobbeurteilung der Resektabilität erfolgt aufgrund von morphologischen Kriterien (hängender, infiltrierender oder verdrängend wachsender Tumor, mögliche Infiltration oder nur Verdrängung von intra- und extrahepatischen Strukturen).

Die formellen, segmentalen und atypischen Leberresektionen erfolgen auf der Basis der chirurgischen Leberanatomie und werden heute vor allem nach der Nomenklatur von Couinaud [5] bezeichnet, nach der die Leber in Sektoren und Segmente unterteilt ist, von denen jedes einzelne Segment eine hepatische Vene und eine portale Triade (Gallenweg, 


\section{Tabelle 1}

Fortschritte der Leberchirurgie.

Präoperative Abklärung mit bildgebenden Verfahren

- Spiral-CT, CT mit Kontrastmittel, MRI, 3D-Rekonstruktion, PET-Scan.

Präoperative Bestimmung der Leberfunktion

- Gaslaktose-Eliminations-Kapazität (GEK), Aminopyrin-Atemtest.

Anästhesie
- tiefer ZVD bei Resektionsphase, früher Ersatz von Plasmafaktoren.

Intensivmedizin

- verzögerte Extubation, Monitoring, Periduralanästhesie.

\section{Onkologische Therapie}

- prä- und postoperative Therapien, regionale Leberperfusion über Port-a-cath.

Chirurgische Verfahren und Techniken

- Ultraschalldissektor, Argonstrahlkoagulator, erweiterte Leberresektionen, segmentale Leberresektionen.

Leberanatomie

- grösseres chirurgisches Verständnis.

\section{Tabelle 2}

Neue Verfahren zur Behandlung von Lebermetastasen.

\section{Perkutane Alkoholinjektion.}

Radiohochfrequenztherapie.*

Kryotherapie.*

Interstitielle Lasertherapie.*

Elektrotherapie.

Regionale Leberperfusion mit Port über die A. gastroduodenalis mit Chemotherapeutika.

* intraoperativ und interventionell perkutan

Arterie, Portalvene) besitzt. Die formellen Resektionen umfassen deshalb folgende Möglichkeiten: monosegmental (jedes einzelne Segment möglich), links lateral (Segmente II und III), Hemihepatektomie links (Segmente II/III/IV), Hemihepatektomie rechts (Segmente V/VI/VII/VIII), erweitert links (zusätzlich Segmente V und VIII), erweitert rechts (zusätzlich Segment IVa und IVb).

Obwohl die Erfahrung und die Hand des Chirurgen auch heute noch die wichtigste Voraussetzung für den Erfolg einer Leberresektion sind, werden verschiedene Geräte und Instrumente für eine blutsparende Leberresektion eingesetzt. Wir verwenden bei unseren Resektionen routinemässig: intraoperative Ultraschalluntersuchung, Ultraschalldissektor, Argonstrahlkoagulator, Elektrokoagulation und Fibrinkleber aus patienteneigenem Serum.

Generell erreicht die chirurgische Leberresektion nach internationalen Serien bei singulären kolorektalen Lebermetastasen unter $5 \mathrm{~cm}$ Durchmesser und einem Resektionsabstand zum Tumor von mindestens einem Zentimeter folgende Resultate: Letalität 0-5\%, Morbidität (Blutung, Galleleck, Leberinsuffizienz) 5-15\%, 5-Jahres-Überlebenszeit 25-45\%. Diese Resultate werden durch verschiedene Faktoren beeinflusst. Iwatsuki et al. [6] fanden bei 243 Patienten folgende negative prognostische Faktoren: einen tumorbefallenen Resektionsrand, extrahepatischen Tumor inklusive Lymphknoten, bilobären Tumorbefall und eine Zeit von der Primäroperation zur Leberresektion von unter 30 Monaten. Eine signifikante Verminderung der Überlebenszeit wurde auch beim Vorliegen von mehr als drei Metastasen und einer Tumorgrösse über $8 \mathrm{~cm}$ gefunden. Fong et al. [7] haben einen Voraussage-Score («Clinical Sore Predicting Recurrence») auf der Basis von 1001 resezierten Patienten entwickelt, in dem folgende Faktoren einen negativen Einfluss auf die Überlebenszeit haben: tumorbefallener Resektionsrand, extrahepatischer Tumor, positive Lymphknoten bei der primären Kolonresektion, Zeit von der Primäroperation zur Leberresektion von unter 12 Monaten, Anzahl der Metastasen mehr als eine, Tumorgrösse über $5 \mathrm{~cm}$ und ein CEA-Titer von über $200 \mathrm{ng} / \mathrm{ml}$. Die berechnete Überlebenszeit für 5 Jahre war 33\% und für 10 Jahre $22 \%$. Kein Patient mit mehr als 5 Faktoren überlebte 5 Jahre. In zirrhotischen Patienten ist die Resektabilität wegen der verminderten Funktion der Restleber oft eingeschränkt und die Komplikationsrate erhöht, wenn auch Midorikawa et al. [8] in einer Serie von 277 Patienten bezüglich der Resektabilität und der postoperativen Komplikationen keine Unterschiede fanden.

Viele andere, grosse Serien sind heute publiziert und bestätigen die Resultate der Leberresektionen. Trotzdem gibt es immer wieder Diskussionen über einzelne prognostische Faktoren wie z.B. die Anzahl der resezierten Metastasen, die noch ein günstiges Überlebensresultat ermöglichen. Diese fundierten Resultate zeigen auch, dass in spezialisierten hepatobiliären Zentren sehr gute Resultate mit Langzeitüberlebenden erreicht werden. Die Indikation zur Leberresektion für kolorektale Lebermetastasen ist deshalb heute für wenige (unter 5) Metastasen sicher gut begründet und sollte den betroffenen Patientinnen und Patienten sicher nicht vorenthalten werden. Eine ganz kritische Frage bezüglich der Resektabilität von Lebermetastasen hat kürzlich Wigmore aufgeworfen. Er stellt nämlich die Frage, ob es einen Unterschied bei der Resektabilität von Patientinnen und Patienten mit Lebermetastasen gibt, wenn die Patienten bei der primären Kolonresektion von einem "general surgeon" oder einem "colorectal specialist" operiert wurden. Er fand eine signifikant bessere Resektabilität der Lebermetastasen und eine signifikant bessere Überlebenskurve für Patienten, bei denen die primäre Operation von "colorectal specialists» durchgeführt worden war.

\section{Neue Verfahren zur Therapie von Lebermetastasen}

Die Leberresektion gilt bisher als einzige Behandlungsmethode mit einer Hoffnung auf Heilung. Die meisten Patientinnen und Patienten sind aber bei der Diagnosestellung von Lebermetastasen mit konventionellen Resektionen bereits nicht mehr kurativ resektabel, da in der Mehrzahl der Fälle ein diffuser, multifokaler oder bilateraler Befall besteht oder zu 
viele Metastasen gefunden werden. In den letzen Jahren wurde deshalb immer wieder nach Methoden gesucht, Lebermetastasen (und auch Lebertumoren) mit nicht resektablen, sogenannt "ablativen", d.h. zerstörenden Behandlungsformen zu bekämpfen. In dieser Absicht wurden in der letzten Zeit die Kälte(Kryotherapie), die Hitze- und die Laserzerstörung von Lebermetastasen und -tumoren mit Erfolg eingesetzt. Diese ablativen Methoden sind in Tabelle 2 aufgeführt.

Die Kryotherapie ist ein seit langem bekanntes Verfahren, das seit kurzem nach der Neuentwicklung von Hochleistungsgeräten mit Schnellgefrierung für die Behandlung von Lebermetastasen eingesetzt wird. Bei der Kryotherapie werden Spezialsonden am offenen Abdomen unter Ultraschallkontrolle oder perkutan unter Kontrolle bildgebender Verfahren in die Tumoren der Leber eingefügt und das Gewebe tiefgefroren. An der Spitze der Sonden werden Temperaturen von bis $\mathrm{zu}-185^{\circ} \mathrm{C}$ erreicht, in der Peripherie des sich bildenden Eisballs wird Tumorgewebe bei Temperaturen bis $-40^{\circ} \mathrm{C}$ vollständig zerstört. Aus physikalischen Gründen können deshalb Tumoren mit einem Durchmesser von bis zu 2,5 cm mit einer Sonde, grössere mit einer Kombination verschiedener Sonden zerstört werden. Es können bis zu sechs Oberflächen- oder Stichsonden parallel eingeführt und vereist werden. Wallace et al. [9] haben in einer Serie von 137 Patienten mit kolorektalen Lebermetastasen in einer Studie von August 1993 bis Juli 1999 eine alleinige Resektion bei 34, eine alleinige Kryotherapie bei 20 und eine kombinierte Therapie von Resektion und Kryotherapie bei 52 Patienten durchgeführt. Bei einer Kontrollzeit von 36 Monaten schliessen die Autoren aus ihren Resultaten, dass das Überleben nur bestimmt wird durch die Zerstörung aller Tumoren. Alter, Art der Therapie und die Anzahl behandelter Metastasen haben bezüglich des Überlebens in dieser Studie keine Rolle gespielt. Sie folgern weiter, dass die Kryotherapie eine Zusatzmethode in der Leberchirurgie sei, und dass Patienten, bei denen die Metastasen wegen ihrer grossen Anzahl als irresektabel angesehen worden sind, einer resektiven bzw. ablativen Therapie mit der Chance auf ein Langzeitüberleben zugeführt werden können. Ähnliche Resultate werden auch von Morris, einem Pionier der Leberkryotherapie, berichtet. Nach seiner Erfahrung mit über 300 kryotherapeutisch behandelten Patienten sollte die 5-Jahres-Überlebenszeit für Patienten mit irresektablen kolorektalen Lebermetastasen wenigstens 20\% sein [10]. Als wichtigste Nebenwirkung der Kryotherapie werden Leberabszesse der zerstörten Gewebe und in etwa 0,3-1\% ein sogenannter Kryoschock beobachtet, bei welchem ein akutes Leber- und Nierenversagen auftritt, das intraoperativ oder unmittelbar postoperativ zum Tode führt und kaum behandelt werden kann [11]. Die Kälte wirkt nicht in der Umgebung von grossen Gefässen, da die Kälte durch den warmen Blutstrom abgeleitet wird. Gallenwege hingegen werden durch die Kälte zerstört oder können Strikturen entwickeln.
Die Hitzetherapie mit einer Radiohochfrequenztherapie zerstört Gewebe durch eine hitzeinduzierte Koagulationsnekrose. Die Hitze wird durch Radiohochfrequenzgeräte erzeugt und über feine Sonden unter Ultraschallkontrolle in das Gewebe eingebracht. An der Spitze dieser Sonden werden mehrere feine Drähte fächerförmig in das Gewebe eingeführt und die Hitze zur Gewebezerstörung erzeugt. Der Durchmesser der Koagulationsnekrose, durch die das Gewebe wirksam zerstört werden kann, liegt bei etwa $2 \mathrm{~cm}$. Gefässwände und Gallengänge werden durch die Hitze etwa in gleichem Rahmen wie bei der Kryotherapie mitkoaguliert.

Izzo et al. [12] haben 304 Patienten mit Radiohochfrequenztherapie behandelt. 40\% Leberzellkarzinome, 46\% kolorektale Lebermetastasen und 14 andere Metastasen der Leber. In 66\% wurde die Hitzetherapie bei offenem Abdomen und in 34\% perkutan eingesetzt. Nach einer nur kurzen Kontrollzeit von median 10 Monaten wurden Rezidivtumoren an der Stelle von behandelten Tumoren in 9\% vor allem bei Tumoren mit einem Durchmesser von mehr als $5 \mathrm{~cm}$ und neue oder extrahepatische Metastasen in $47 \%$ der Patienten gefunden. Die Letalität betrug $0,3 \%$.

Die interstitielle Lasertherapie der Lebermetastasen wird heute in kontrollierten Studien untersucht, ist abhängig von teilweise sehr aufwendigen Geräten und zurzeit noch wenig klinisch eingesetzt. Neuere Geräte mit feinsten Stichsonden für offene, laparoskopische oder perkutane Verfahren sind aber in Entwicklung. Wir haben mit diesen Lasertherapien keine persönlichen Erfahrungen.

In der klinischen Erprobung sind Verfahren, bei denen das Tumorgewebe durch Anlage einer elektrischen Spannung zerstört werden sollen. Der Zelltod tritt bei dieser Methode durch eine intrazelluläre Verschiebung von Elektrolyten und der damit verbundenen Zerstörung der Zellstruktur ein.

Wo der Stellenwert der verschiedenen Methoden heute liegt, und welche Methode in welcher Situation angewendet werden soll, kann heute noch nicht abschliessend beurteilt werden. Es scheint aber, als ob die Radiohochfrequenztherapie bei Tumoren unter $2 \mathrm{~cm}$ Durchmessern einen gewissen Vorteil gegenüber der Kryotherapie hat (kein Kryoschock), dass die Kryotherapie aber wahrscheinlich eine komplettere Zerstörung von grösseren Tumoren erreichen kann. Wir sind der Meinung, dass bei operablen Patienten diese Verfahren erstmals bei einer chirurgischen Revision der Leber nach vollständiger Mobilisation und intraoperativer Prüfung des Befundes durch Ultraschall eingesetzt werden sollen. Nur bei einer chirurgischen Revision lässt sich unserer Meinung nach heute abschliessend beurteilen, ob eine Resektion möglich ist und ob Tumoren vorliegen, die in den bildgebenden Methoden aus Gründen der heutigen Auflösungsgrenzen nicht gesehen werden können. Nur bei inoperablen Patienten und beim Auftreten von Rezidiven nach Resektion sollen die Tumoren direkt perkutan behandelt werden. 
Welche Resultate schliesslich mit den neuen Verfahren erreicht werden können, ist heute noch nicht ganz klar. Ob eine singuläre Lebermetastase alleine mit einem neuen Verfahren ohne Resektion behandelt werden darf, ist ebenfalls eine offene Frage. In Deutschland wird zurzeit immerhin die Diskussion über diese Frage geführt, ob es ethisch vertretbar sei, eine vergleichende Studie zwischen ablativen und resezierenden Verfahren für kolorektale singuläre Metastasen multizentrisch durchzuführen. Vor einem Jahr wäre diese Idee nur belächelt worden.

Wir sind der Meinung, dass heute aufgrund der vorliegenden ersten Resultate der ablativen Verfahren für die offene oder perkutane Behandlung von multiplen sogenannt irresektablen kolorektalen Metastasen eine gute Indikation besteht. Diese ablativen Verfahren kombiniert mit einer Leberresektion können die bisherigen konservativen Indikationsstellungen erweitern und bisher als irresektabel beurteilte Patienten neu einer chirurgischen Therapie zuführen. Wir müssen uns aber weiter darüber im klaren sein, dass die heutigen resezierenden und ablativen Therapien lokale Behandlungsverfahren sind, die für eigentlich disseminierte oder wenigstens multifokale Krankheiten eingesetzt werden, weil noch keine ursächliche therapeutische Möglichkeit besteht.

\section{Zusammenfassung}

Die Leberresektion für singuläre oder unilaterale kolorektale Lebermetastasen ist heute eine etablierte Behandlungsmethode, die in den Händen von hepatobiliären Chirurgen gute Resultate mit einer Letalität von $0-5 \%$, einer Morbidität von 5-15\% und ein 5-Jahres-Überleben von 25-45\% erreicht. Diese Erfolge in der Behandlung von Lebermetastasen werden nur in interdisziplinärer Zusammenarbeit mit interessierten hepatobiliären Spezialisten im Team erreicht.
Die kürzliche Einführung von lokal zerstörenden Methoden der Kälte-, Hitze- oder Lasertechnik zur alleinigen oder kombinierten offenen und perkutanen Behandlung von Lebermetastasen zeigt erste ermutigende Resultate und erweitert heute die Indikation zur Behandlung von Lebermetastasen und Lebertumoren.

\section{Literatur}

1 Fortner JG, Silva JS, Golbey RB, Cox EB, Maclean BJ. Multivariate analysis of a personal series of 247 consecutive patients with liver metastases from colorectal cancer. I. Treatment by hepatic resection. Ann Surg 1984;199:306-16.

2 Adson MA. Resection of liver metastases - when is it worthwhile? World J Surg 1987;11:511-20.

3 Fong Y, Blumgart LH, Cohen AM. Surgical treatment of colorectal metastases to the liver. CA Cancer J Clin 1995; 45:50-62.

4 Bengtsson G, Carlsson G, Hafstom L, Johnsson PE. Natural History of patients with untreated liver metastases from colorectal cancer. Am J Surg 1981;141:586-9.

5 Couinaud C. Controlled hepatectomies and exposure of the intrahepatic bile ducts. Paris; 1981.

6 Iwatsuki S, Dvorchik I, Madariaga JR, Marsh JW, Dodson F, Bonham AC, et al. Hepatic resection for metastatic colorectal adenocarcinoma: a proposal of a prognostic scoring system. J Am Coll Surg 1999;189:291-9.

7 Fong Y, Fortner J, Sun RL, Brennan MF, Blumgart LH. Clinical score for predicting recurrence after hepatic resection for metastatic colorectal cancer: analysis of 1001 consecutive cases. Ann Surg 1999;230:309-21.

8 Midorikawa Y, Kubota K, Takayama T, Toyoda H, Ijichi M, Torzilli G, et al. A comparative study of postoperative complications after hepatectomy in patients with and without chronic liver disease. Surgery 1999;126:484-91.

9 Wallace JR, Christians KK, Pitt HA, Quebbeman EJ. Cryotherapy extends the indications for treatment of colorectal liver metastases. Surgery 1999;126:766-74.

10 Morris LM. Results of hepatic cryotherapy. In: $4^{\text {th }}$ World Congress oft IHPBA 2000. Handbook and Abstracts.

11 Feifel G, Schüder G, Pistorius G. Kryochirurgie - Renaissance oder echter Fortschritt? Chirurg 1999;70:154-9.

12 Izzo F, Delrio P, Ellis L, Woodall M, Marra P, Wolff R, et al. Radiofrequency ablation of malignant tumours in 304 patients. In: $4^{\text {th }}$ World Congress oft IHPBA 2000. Handbook and Abstracts. 